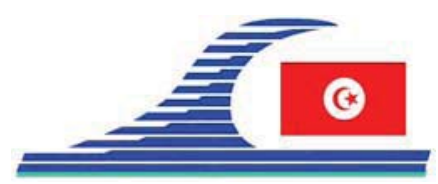

\title{
Fudaa-Albe : Analyse élasto-plastique des Ducs d'Albe selon une approche semi-probabiliste
}

\section{Claire GUIZIOU ${ }^{1}$, Olivier SOULAT ${ }^{2}$, Jean-Jacques TRICHET ${ }^{1}$}

1. CETMEF, Centre d'Etudes Techniques Maritimes et Fluviales, 2 bd Gambetta, BP 60038, 60321 Compiègne Cedex, France.

claire.guiziou@developpement-durable.gouv.fr

jean-jacques.trichet@developpement-durable.gouv.fr

2. Grand Port Maritime de Marseille,

23 place de la Joliette, BP 81965, 13226 Marseille Cedex 02, France.

olivier.Soulat@marseille-port.fr

\section{Résumé :}

L'élaboration des Eurocodes, normes européennes sur le calcul des constructions, a été menée depuis les années 80. Ils sont basés sur les méthodes semi-probabilistes qui introduisent des coefficients partiels. Actuellement, peu de logiciels sont capables de prendre en compte cette approche semi-probabiliste. Ils doivent s'adapter pour répondre aux enjeux et critères des nouvelles normes européennes.

Partant du constat que peu de logiciels permettent le calcul semi-probabiliste des Ducs d'Albe, le CETMEF a décidé de développer un logiciel : Fudaa-Albe (CETMEF, site web). Ce logiciel est basé sur l'utilisation du logiciel Albe développé dans les années 80 par le CETMEF, permettant le dimensionnement par analyse élasto-plastique des Ducs d'Albe avec une approche globale. Ce logiciel a été modifié pour intégrer une approche semi-probabiliste et complété par l'utilisation d'une nouvelle interface de calcul: Fudaa qui facilite son utilisation (logiciel autrefois développé sous DOS).

Les défenses d'accostage et le calcul d'énergie d'accostage sont intégrés en données d'entrée du logiciel et donc pris en compte dans le calcul pour faciliter le travail du concepteur.

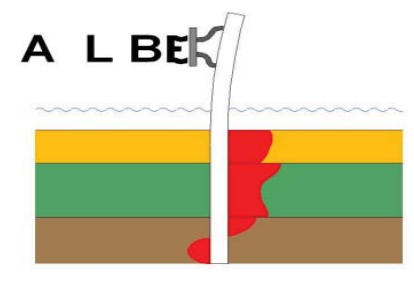

\section{Mots-clés :}

Ducs d'Albe - Analyse élasto-plastique - approche semi-probabiliste - Eurocodes Logiciel. 


\section{Introduction}

ALBE est un logiciel développé au cours des années 1980 (CETMEF, 1990). Ce logiciel permet de calculer les ducs d'Albe d'accostage et d'amarrage par une méthode élasto-plastique (ou aux coefficients de réaction). L'accostage est modélisé par une énergie, Albe calcule les efforts et déplacements du pieu correspondant à l'absorption de cette énergie en prenant en compte la présence éventuelle d'une défense. L'amarrage est modélisé par une force statique en tête de pieu. Il permet de calculer les efforts et déplacements résultant de ce calcul.

A notre connaissance il n'existerait pas de logiciels dans le commerce permettant le calcul des Ducs d'Albe d'accostage. Seuls seraient commercialisés des logiciels pour le calcul de pieux soumis à un effort horizontal en tête (pour l'amarrage).

Les méthodes semi-probabilistes (ou aux coefficients partiels) deviennent d'utilisation systématique en Europe avec la mise en application des Eurocodes, des annexes nationales et des normes d'application nationales (en cours de rédaction). Les Recommandations pour le calcul aux états-limites des Ouvrages en Site Aquatique (ROSA 2000), (CETMEF, 2001) destinées à préparer à la mise en œuvre des méthodes semi-probabilistes illustrent que l'utilisation de coefficients partiels va profondément bouleverser la façon de mener les calculs.

Le choix a été fait de développer ce logiciel à la suite de la diffusion de ROSA 2000 (CETMEF, 2001) pour répondre à un besoin d'adaptation des logiciels aux Eurocodes.

\section{Données d'entrée du logiciel}

\subsection{Principe général du calcul}

Par un calcul itératif, le programme cherche à déterminer la position d'équilibre sous chargement du pieu, dont la base est la résolution par la méthode des différences finies d'une équation différentielle du quatrième ordre (CETMEF, 1990).

Le pieu est découpé en $n$ tronçons pour lesquels les caractéristiques du pieu et de sol sont constantes. Chaque tronçon de sol est considéré comme étant appuyé sur un ressort dont la courbe réaction/écrasement (ou allongement) est non-linéaire. Cette courbe correspond à la courbe de réaction du sol.

La solution d'équilibre est déterminée lorsque la différence de déplacement en plusieurs points du pieu entre deux itérations successives est inférieure à la tolérance fixée dans le programme de calcul. En plus de ce calcul itératif sur le sol qui a pour base un effort horizontal en tête (action d'amarrage), le programme itère sur cet effort jusqu'à ce que l'énergie absorbée par le tube plus l'énergie absorbée par la défense atteignent la valeur désirée (action d'accostage). 
2.2 Spécificités du logiciel Fudaa-Albe

- Introduction et visualisation des courbes de réaction du sol :

Le logiciel Fudaa-Albe calcule automatiquement les courbes d'interaction élastoplastique (METT, 1993) à partir des données pressiométriques des sols. Il est possible de définir un comportement du sol de longue, courte ou très courte durée, mais aussi de le définir manuellement.

- La géométrie du pieu :

Le pieu peut être décomposé en tronçons : jusqu'à 100 tronçons en prenant en compte diamètre, épaisseur de l'acier et épaisseur sacrificielle pour la corrosion. Cela permet d'optimiser les caractéristiques géométriques du pieux en réduisant l'épaisseur ou la nuance de l'acier sur certaines parties pour optimiser les coûts.

- Les spécificités pour les Ducs d'Albe d'accostage :

Pour ces Ducs d'Albe, Fudaa-Albe permet l'introduction des courbes de réaction des défenses (qu'on peut retrouver dans les catalogues de constructeurs de défenses) pour optimiser au mieux l'ouvrage.

Les navires sont également pris en compte pour calculer les efforts d'accostage à reprendre par les pieux. Le calcul des énergies se fait à partir des caractéristiques de navire: longueur, largeur, tirant d'eau, vitesse d'accostage et des divers coefficients correcteurs attachés à ce calcul $(\mathrm{Ce}, \mathrm{Cm}, \mathrm{Cc}$, et $\mathrm{Cs})$.

\section{L'approche semi-probabiliste}

\subsection{Principe de la méthode: introduction de coefficients partiels}

La méthode de calcul semi-probabiliste (ou aux coefficients partiels) permet de tenir compte de l'incertitude sur les valeurs des résistances des matériaux constituant l'ouvrage, sur les valeurs des paramètres du sol, sur les courbes de comportement des défenses, sur les actions, sur les modèles de calcul utilisés (utilisation de coefficients de modèle conformément à ROSA 2000).

\subsection{La démarche ROSA 2000}

Le logiciel Fudaa-Albe reprend le formalisme et les notions introduits par ROSA 2000. On définit :

- des situations de projet (durables, transitoires, accidentelle...),

- des coefficients partiels à appliquer aux valeurs représentatives des paramètres,

- des combinaisons d'action (ELU fondamental, ELU accidentel, ELS fréquent...),

- des états limites introduits par l'intermédiaire des critères de dimensionnement dans le logiciel: Résistance en flexion du pieu, déplacement et réaction en tête, mobilisation de la butée, déflexion de la défense. 


\subsection{Fudaa-Albe : un logiciel adapté à l'approche semi-probabiliste}

Aujourd'hui peu de logiciels sont adaptés au grand nombre de combinaison d'action à calculer pour lesquels plusieurs états limites peuvent être à vérifier avec l'approche semi-probabiliste. Fudaa-Albe permet par l'intermédiaire de son interface d'introduction de données de définir en amont du calcul tous les coefficients partiels qui seront nécessaires pour la formulation de l'ensemble des combinaisons et états limites. Un seul fichier de calcul est donc à créer, on réduit par conséquent le nombre de fichiers et donc les risques de saisies non cohérentes ainsi que le temps de travail.

Enfin une fois les calculs terminés, le logiciel synthétise l'ensemble des résultats obtenus dans des tableaux : le logiciel compile les facteurs de dimensionnement pour chaque état limite de chaque combinaison (rapport entre "l'élément " résistant et « l'élément» mobilisé pour un état limite et un modèle de calcul donnés). Quand ce facteur est inférieur à 1, la structure est sous-dimensionnée, quand il est nettement supérieur à 1 , une optimisation de la structure est à envisager. Une exportation des résultats obtenus est possible pour une exploitation sous tableur.

Enfin, le logiciel permet de générer ou exporter des notes de calcul synthétisant les résultats et les combinaisons que le concepteur veut retenir pour une meilleure lisibilité du projet.

\section{Conclusions}

Le logiciel Fudaa-Albe développé par le CETMEF pour le calcul élasto-plastique des Ducs d'Albe vient apporter un outil au concepteur de projet adapté au dimensionnement selon le format semi-probabiliste introduit dans les Eurocodes. Il permet d'intégrer des données (défenses, navires, ...) spécifiques aux ouvrages d'accostage tout en permettant une analyse synthétique des résultats pour les différents états-limites à considérer et des nombreuses combinaisons d'action à envisager pour faciliter le travail du concepteur. L'approche Eurocode permet de rechercher une optimisation dans le dimensionnement de ces ouvrages par rapport aux méthodes dites « classiques ».

\section{Références}

CETMEF (1990). Notice d'utilisation du logiciel Albe. Notice STC INF 90.13, CETMEF Compiègne, $43 \mathrm{p}$.

CETMEF (2001). Rosa 2000 - Recommandations pour le calcul aux états-limites des ouvrages en site aquatique. CETMEF - Pont-Formation Éditions, environ 2000 p.

CETMEF (site web). Fudaa-Albe. Logiciel téléchargeable librement en ligne sur [ http://www.cetmef.developpement-durable.gouv.fr/portuaire/fudaa-albe.html ] METT -Ministère de l'Équipement, des Transports et du Tourisme (France)- (1993). Règles techniques de conception et de calcul des fondations des ouvrages de génie civil. Fascicule $n^{\circ} 62$, Titre V. Direction des journaux officiels. 